\title{
A UNIFORMLY CONVERGENT METHOD FOR A SINGULARLY PERTURBED SEMILINEAR REACTION-DIFFUSION PROBLEM WITH MULTIPLE SOLUTIONS
}

\author{
GUANGFU SUN AND MARTIN STYNES
}

\begin{abstract}
This paper considers a simple central difference scheme for a singularly perturbed semilinear reaction-diffusion problem, which may have multiple solutions. Asymptotic properties of solutions to this problem are discussed and analyzed. To compute accurate approximations to these solutions, we consider a piecewise equidistant mesh of Shishkin type, which contains $O(N)$ points. On such a mesh, we prove existence of a solution to the discretization and show that it is accurate of order $N^{-2} \ln ^{2} N$, in the discrete maximum norm, where the constant factor in this error estimate is independent of the perturbation parameter $\varepsilon$ and $N$. Numerical results are presented that verify this rate of convergence.
\end{abstract}

\section{INTRODUCTION}

Singularly perturbed nonlinear boundary value problems occur frequently in engineering applications such as catalytic reactions or absorption processes and fluid dynamics; see Smith [16, Chapter 10].

We consider the semilinear problem

$$
F_{\varepsilon} u(x) \equiv-\varepsilon^{2} u^{\prime \prime}(x)+b(x, u)=0 \quad \text { for } x \in(0,1),
$$

$$
u(0)=u(1)=0,
$$

where $\varepsilon$ is a small positive parameter. Set $X=[0,1]$. We shall assume that $b \in C^{\infty}\left(X \times \mathcal{R}^{1}\right)$ for convenience.

Asymptotic and numerical solutions of problem (1.1) have been considered by many authors, under various hypotheses on $b(x, u)$. See for example Chang and Howes [1], D'Annunzio [2], Fife [4], Herceg [7], Herceg and Petrović [8] and Lorenz [9].

One of the conditions occurring frequently in the literature is

$$
b_{u}(x, u)>b_{0}^{2}>0 \text { for all }(x, u) \in X \times \mathcal{R}^{1} .
$$

Under this condition, problem (1.1) has a unique solution $u \in C^{\infty}(X)$; see Lorenz [9].

The reduced problem of (1.1) is defined by

$$
b\left(x, u_{0}\right)=0 \quad \text { for } x \in X .
$$

Received by the editor December 16, 1993 and, in revised form, April 3, 1995.

1991 Mathematics Subject Classification. Primary 34E15, 65L10, 65L12, 65L50.

(C)1996 American Mathematical Society 
Under condition (1.2), this reduced problem has a unique solution $u_{0} \in C^{\infty}(X)$, as can be seen using the implicit function theorem and the compactness of $X$. Note that in general, $u_{0}$ does not satisfy either of the boundary conditions in (1.1b).

The reduced problem (1.3) may have more than one solution if condition (1.2) is not satisfied. Fife [4], D'Annunzio [2] and O'Malley [11] consider problem (1.1) under the assumptions that:

(i) it has a stable reduced solution, i.e., there exists a solution $u_{0} \in C^{\infty}(X)$ of (1.3) such that

$$
b_{u}\left(x, u_{0}\right)>b_{0}^{2}>0 \quad \text { for all } x \in X
$$

(ii) it has stable boundary layers, i.e., the stable reduced solution $u_{0}$ of (i) satisfies

$$
\int_{u_{0}(0)}^{\tau} b(0, s) d s>0 \text { for }\left\{\begin{array}{l}
\tau \in\left(u_{0}(0), 0\right], \text { whenever } 0>u_{0}(0), \\
\tau \in\left[0, u_{0}(0)\right), \text { whenever } u_{0}(0)>0,
\end{array}\right.
$$

and

$$
\int_{u_{0}(1)}^{\tau} b(1, s) d s>0 \text { for }\left\{\begin{array}{l}
\tau \in\left(u_{0}(1), 0\right], \text { whenever } 0>u_{0}(1), \\
\tau \in\left[0, u_{0}(1)\right), \text { whenever } u_{0}(1)>0 .
\end{array}\right.
$$

The conditions (1.4) are obviously weaker than condition (1.2). Condition (1.4a) implies that any solution of (1.3) is locally unique. Problem (1.1) under the conditions (1.4) may exhibit multiple solutions. D'Annunzio [2] uses degree theory to prove existence and local uniqueness of a solution satisfying (1.1) and (1.4).

In what follows, we shall refer to (1.1) under condition (1.2) as problem (A) and (1.1) under conditions (1.4) as problem (B).

A solution $u(x)$ of (1.1) usually exhibits sharp boundary layers at the endpoints of the interval $X$ when the parameter $\varepsilon$ is near zero. When polynomial-based numerical methods are applied to (1.1), one does not obtain accurate results on all of $X$, even in the linear case. This has led to the development of numerical methods that are uniformly convergent with respect to the perturbation parameter.

Let $u$ be a solution of (1.1). Consider a difference scheme for solving (1.1). Suppose that this scheme has a solution $u^{N}$ that satisfies

$$
\left\|u-u^{N}\right\| \leq C g(N),
$$

where $N$, independent of $\varepsilon$, is the number of subintervals in the mesh used, $C$ is a positive constant independent of $N$ and $\varepsilon$, and $g(N)$ is a function of $N$ but is independent of $\varepsilon$. If $g(N) \rightarrow 0$ as $N \rightarrow \infty$, then we say that the scheme is uniformly convergent to $u$, with respect to the norm $\|\cdot\|$. Furthermore, we shall say that the scheme is uniformly convergent with order $g(N)$ in the norm $\|\cdot\|$. In this paper, we consider only uniform convergence with respect to the discrete $L^{\infty}$ norm.

In the linear case, many authors consider both uniformly convergent exponentially fitted schemes on equidistant meshes and uniformly convergent polynomialbased schemes on special meshes; see Doolan et al. [3], Hegarty et al. [6], Niijima [10], O'Riordan and Stynes [12], Roos [14] and Vulanović [18].

Uniformly convergent methods for the semilinear problem (A) have also been examined. Vulanović [17] applies a central difference scheme to the semilinear problem (A). He obtains second-order uniform convergence of the scheme on a special graded mesh of Bakhvalov type. Herceg [7] investigates a scheme for problem (A) under 
extra somewhat restrictive conditions on the problem. He achieves fourth-order uniform convergence of the scheme, again on a graded mesh of Bakhvalov type.

D'Annunzio [2] uses a simple central difference scheme on a special locally quasi-equidistant mesh to solve the more general problem (B). This mesh contains $O\left(h^{-1} \ln 1 / \varepsilon\right)$ mesh points when $\varepsilon \leq h$, where $h$ is the maximum mesh spacing over the interval $X$. She shows existence of a solution to the discrete problem and $O(h)$ convergence of this solution to a solution of problem (B). The constant factor in the error estimate is independent of $\varepsilon$. The method is not however uniformly convergent in the sense of (1.5), since the number of mesh points depends on the perturbation parameter $\varepsilon$.

In the present paper, we consider a uniformly convergent method for problem (B). This method is D'Annunzio's scheme on a special piecewise equidistant mesh. Such meshes, which were recently introduced by Shishkin [15], are much simpler than the graded meshes of Vulanović [17], Herceg [7] and D'Annunzio [2]. We use degree theory to prove existence of a solution to the scheme. We construct super and sub solutions that are within $O\left(\varepsilon^{2} \ln ^{2}(1 / \varepsilon)\right)$ of a solution of problem (B); we also consider their discrete analogues for the scheme. Then we deduce uniform convergence of $O\left(N^{-2} \ln ^{2} N\right)$ for the scheme under the nonrestrictive assumption that $\varepsilon \leq N^{-1}$. This result is a significant improvement over the first-order convergence obtained by D'Annunzio [2] for the same scheme on a different mesh.

A summary of the paper is as follows. Section 2 contains results concerning the exact solutions of problem (B). In $\S 3$, we bound truncation errors of the central difference scheme on a piecewise equidistant Shishkin mesh. In $\S 4$, we prove existence of solutions and the almost second-order uniform accuracy of the scheme for problem (B). Section 5 presents numerical computations that confirm our results.

Notation. Throughout this paper we let $C$, sometimes subscripted, denote a generic positive constant that may take different values in different formulas, but is always independent of $N$ and $\varepsilon$.

\section{The Continuous PRoblem}

In this section, we discuss the properties of exact solutions of problem (B). We shall suppose, without loss of generality, that $u_{0}(0)<0$ and $u_{0}(1)<0$, as other cases can be handled similarly.

The concepts of super and sub solutions are important for the study of problem (B). Suppose that there exist two functions $\alpha$ and $\beta \in C^{2}(X)$ with the following properties:

$$
\begin{aligned}
F_{\varepsilon} \alpha(x) & \leq 0 \leq F_{\varepsilon} \beta(x) \quad \text { for } x \in X, \\
\alpha(0) & \leq 0 \leq \beta(0), \\
\alpha(1) & \leq 0 \leq \beta(1), \\
\alpha(x) & \leq \beta(x) \quad \text { for } x \in X .
\end{aligned}
$$

Then $\beta(x)$ and $\alpha(x)$ are said to be super and sub solutions respectively of problem (B).

In order to prove higher-order convergence of a central difference scheme for problem (B), we shall introduce super and sub solutions that are more accurate than those in D'Annunzio [2]. Let us first give some notation and definitions. 
We use a cutoff function $\sigma(x)$ defined by

$$
\sigma(x)= \begin{cases}1 & \text { for } 0 \leq x \leq 1 / 4 \\ 0 & \text { for } 1 / 2 \leq x \leq 1\end{cases}
$$

where $\sigma \in C^{\infty}(X)$ and $\sigma$ is monotonically decreasing.

Let $v \in C^{\infty}(0, \infty)$. Let $J$ denote a positive integer. Let $b_{1}$ be a positive constant. If for each $\delta \in\left(0, b_{1}\right)$ there exists a positive constant $C_{\delta}$, depending on $\delta$ and $J$, such that

$$
\left|v^{(j)}(\eta)\right| \leq C_{\delta} \exp \left(-\left(b_{1}-\delta\right) \eta\right)
$$

for $\eta>0$ and $j=0,1, \ldots, J$, then we say that the function $v(\eta)$ belongs to the class $e\left(b_{1}, J\right)$.

In the rest of this section, we shall use $J$ to denote an arbitrary positive integer. In fact, we shall take $J=4$ in the analysis of $\S \S 3$ and 4 .

The following two lemmas are modifications of Lemmas 2.1 and 2.2 of Fife [4].

Lemma 2.1. Let $\lambda>0$ be a constant. Let $g \in C^{\infty}[0, \infty)$ satisfy $g(0)=0, g^{\prime}(0)>0$ and

$$
\int_{0}^{\tau} g(s)>0 \quad \text { for } \tau \in(0, \lambda] .
$$

Then for $\eta \geq 0$, there exists a unique strictly decreasing solution $v(\eta)$ of

$$
\begin{aligned}
& v^{\prime \prime}-g(v)=0 \quad \text { for } \eta>0, \\
& v(0)=\lambda, \quad v(\infty)=0
\end{aligned}
$$

Furthermore, $v$ belongs to the class $e\left(b_{1}, J\right)$ with $b_{1}=\sqrt{g^{\prime}(0)}$.

Proof. By Lemma 2.1 of Fife [4], the solution $v$ of (2.1) and (2.2) exists, is strictly decreasing and satisfies

$$
C_{\delta}^{-1} \exp \left(-\left(b_{1}+\delta\right) \eta\right) \leq v^{(j)}(\eta) \leq C_{\delta} \exp \left(-\left(b_{1}-\delta\right) \eta\right),
$$

for $j=0,1$ and $\eta>0$, where $b_{1}=\sqrt{g^{\prime}(0)}, \delta \in\left(0, b_{1}\right)$ and $C_{\delta}>0$ are constants.

Since $g(0)=0$ and $g^{\prime}(s)$ is bounded for $s \in(0, \lambda)$, we have from $(2.1)$

$$
\begin{aligned}
\left|v^{\prime \prime}(\eta)\right| & =\left|g^{\prime}\left(v^{*}\right)\right| v(\eta), \quad \text { where } v^{*} \in(0, v) \subseteq(0, \lambda), \\
& \leq C_{\delta} \exp \left(-\left(b_{1}-\delta\right) \eta\right) \quad \text { for } \eta>0
\end{aligned}
$$

where we recall that $C_{\delta}$ is a generic constant. The result then follows from differentiating (2.1) repeatedly and induction on $j$, since the derivatives of $g(s)$ up to any prescribed order are bounded for $s \in(0, \lambda)$.

Lemma 2.2. Let $\lambda$ and $g(s)$ be as in Lemma 2.1. Let $v(\eta)$ be the strictly decreasing solution of (2.1) and (2.2). Let $a(\eta)$ belong to the class $e\left(b_{1}, J\right)$ with $b_{1}=\sqrt{g^{\prime}(0)}$, and let $\lambda_{1}$ be a given constant. Then there exists a unique solution $v_{1}(\eta)$ of

$$
\begin{aligned}
& v_{1}^{\prime \prime}(\eta)-g^{\prime}(v(\eta)) v_{1}(\eta)=a(\eta) \quad \text { for } \eta>0 \\
& v_{1}(0)=\lambda_{1}, \quad v_{1}(\infty)=0 .
\end{aligned}
$$

Moreover, $v_{1}(\eta)$ belongs to the class $e\left(b_{1}, J\right)$.

Proof. The result follows easily from an inspection of the proof of Lemma 2.2 in Fife [4].

The next lemma is a modification of Lemma 3.1 of D'Annunzio [2]. 
Lemma 2.3. Let $\lambda$ and $g(s)$ be as in Lemma 2.1. Let $p$ be a constant. Then there is a $p_{0} \in\left(0, g^{\prime}(0)\right)$ such that if $|p|<p_{0}$, there exists a unique solution $v(\eta, p)$ of

$$
\begin{aligned}
& \ddot{v}-g(v)=-p v \quad \text { for } \eta>0, \\
& v(0, p)=\lambda, \quad v(\infty, p)=0 .
\end{aligned}
$$

(Here and in what follows a dot denotes partial differentiation with respect to $\eta$.) For each fixed $p \in\left(-p_{0}, p_{0}\right)$, the solution $v(\eta, p)$ is strictly decreasing in $\eta$ and belongs to the class $e\left(b_{1}, J\right)$, where $b_{1}=\sqrt{g^{\prime}(0)-p}$.

The derivative $\phi(\eta, p)=\frac{\partial v}{\partial p}(\eta, p)$ exists and satisfies

$$
\begin{aligned}
& \ddot{\phi}-\left(g^{\prime}(v)-p\right) \phi=-v \quad \text { for } \eta>0, \\
& \phi(0, p)=\phi(\infty, p)=0
\end{aligned}
$$

and

$$
\phi(\eta, p)>0 \quad \text { for } \eta>0 .
$$

Furthermore, there exist positive constants $C_{1}$ and $C_{2}$, independent of $p$, such that

$$
|\phi(\eta, p)| \leq C_{1} \eta e^{-C_{2} \eta} \quad \text { for }(\eta, p) \in(0, \infty) \times\left[-p_{0}, p_{0}\right] .
$$

Proof. The argument is similar to that of Lemma 3.1 of D'Annunzio [2]. We give an outline of the proof for completeness.

Let $g_{p}(s)=g(s)-p s$. We can easily show that there exists a $p_{0} \in\left(0, g^{\prime}(0)\right)$ such that if $|p|<p_{0}$, then $g_{p}(s)$ satisfies the conditions on $g(s)$ in Lemma 2.1. Therefore, the problem $(2.3)-(2.4)$ has a unique solution $v(\eta, p)$. For each fixed $p \in\left(-p_{0}, p_{0}\right)$, the solution $v(\eta, p)$ is strictly decreasing in $\eta$ and belongs to the class $e\left(b_{1}, J\right)$ with $b_{1}=\sqrt{g^{\prime}(0)-p}$.

Also, from the proof of Lemma 2.1, we see that

$$
C_{\delta}^{-1} \exp \left(-\left(b_{1}+\delta\right) \eta\right) \leq \frac{\partial^{j} v}{\partial \eta^{j}}(\eta, p) \leq C_{\delta} \exp \left(-\left(b_{1}-\delta\right) \eta\right),
$$

for $j=0,1$ and $\eta>0$, where $\delta \in\left(0, b_{1}\right)$ and $C_{\delta}>0$ are constants.

Moreover, we can show that $v(\eta, p)$ is differentiable with respect to $p$ and satisfies $\left.\frac{\partial v}{\partial p}(\eta, p)\right|_{\eta=0}=0$. Set

$$
\phi(\eta, p)=\frac{\partial v}{\partial p}(\eta, p) \quad \text { and } \quad \psi(\eta, p)=-\frac{\partial v}{\partial \eta}(\eta, p) .
$$

Then $\phi(\eta, p)$ satisfies (2.5) and (2.6), while $\psi(\eta, p)$ satisfies the homogeneous version of (2.5). Using the method of variation of parameters, we obtain

$$
\phi(\eta, p)=-\psi(\eta, p) \int_{0}^{\eta} \psi^{-2}(\xi, p) \int_{\xi}^{\infty}[-v(\zeta, p)] \psi(\zeta, p) d \zeta d \xi .
$$

Now (2.7) follows from $v(\eta, p)>0$ and $\psi(\eta, p)>0$. Using (2.9) and (2.10), we conclude that (2.8) holds.

We now define the required boundary layer functions. These are more accurate than those of D'Annunzio. They will be used to construct our super and sub 
solutions. Let

$$
w(x, \varepsilon, p)=\left\{\begin{array}{r}
\left(v_{0}^{0}(x / \varepsilon, p)+\varepsilon v_{1}^{0}(x / \varepsilon)\right) \sigma(x) \text { for } 0 \leq x \leq 1 / 2, \\
\left(v_{0}^{1}((1-x) / \varepsilon, p)+\varepsilon v_{1}^{1}((1-x) / \varepsilon)\right) \sigma(1-x) \\
\text { for } 1 / 2<x \leq 1,
\end{array}\right.
$$

where $v_{0}^{0}(\eta, p), v_{0}^{1}(\eta, p), v_{1}^{0}(\eta)$, and $v_{1}^{1}(\eta)$ are respectively defined by

$$
\begin{aligned}
& \ddot{v}_{1}^{0}-b_{u}\left(0, u_{0}(0)+v_{0}^{0}(\eta, 0)\right) v_{1}^{0} \\
& =\eta\left[b_{x}\left(0, u_{0}(0)+v_{0}^{0}(\eta, 0)\right)+b_{u}\left(0, u_{0}(0)+v_{0}^{0}(\eta, 0)\right) u_{0}^{\prime}(0)\right] \text { for } \eta>0, \\
& v_{1}^{0}(0)=0, \quad v_{1}^{0}(\infty)=0,
\end{aligned}
$$

and

$$
\begin{aligned}
& \ddot{v}_{1}^{1}-b_{u}\left(1, u_{0}(1)+v_{0}^{1}(\eta, 0)\right) v_{1}^{1} \\
& =\eta\left[b_{x}\left(1, u_{0}(1)+v_{0}^{1}(\eta, 0)\right)+b_{u}\left(1, u_{0}(1)+v_{0}^{1}(\eta, 0)\right) u_{0}^{\prime}(1)\right] \quad \text { for } \eta>0, \\
& v_{1}^{1}(0)=0, \quad v_{1}^{1}(\infty)=0 .
\end{aligned}
$$

We remark that D'Annunzio uses only the first terms of our expansions, i.e., $v_{1}^{0} \equiv$ $v_{1}^{1} \equiv 0$ in $[2]$.

Lemmas 2.1-2.3 imply that there exists $p_{0}>0$, independent of $\varepsilon$, such that $w(x, \varepsilon, p)$ is well defined for $|p|<p_{0}$. The function $w$ essentially models boundary layers at $x=0$ and $x=1$, as we shall see in the course of proving the next lemma.

Lemma 2.4. Set $p_{\varepsilon}=\varepsilon^{2} \ln ^{2}(1 / \varepsilon)$. Then we can choose positive constants $C_{1}$ and $C_{2}$, which are independent of $\varepsilon$, such that when $\varepsilon$ is sufficiently small, $w\left(x, \varepsilon, C_{1} p_{\varepsilon}\right)$ and $w\left(x, \varepsilon,-C_{1} p_{\varepsilon}\right)$ are well defined, and

$$
\beta(x, \varepsilon)=u_{0}(x)+w\left(x, \varepsilon, C_{1} p_{\varepsilon}\right)+C_{2} p_{\varepsilon}
$$

and

$$
\alpha(x, \varepsilon)=u_{0}(x)+w\left(x, \varepsilon,-C_{1} p_{\varepsilon}\right)-C_{2} p_{\varepsilon}
$$

are super and sub solutions respectively of problem (B).

Proof. Fix $\varepsilon \in(0,1]$. We shall specify $C_{1}$ and $C_{2}$ later in the proof. It is easy to see from (2.7) that

$$
\alpha(x, \varepsilon)<\beta(x, \varepsilon) \quad \text { for } x \in X .
$$

By the construction of $w(x, \varepsilon, p)$, we have

$$
\begin{aligned}
& \alpha(0, \varepsilon)=-C_{2} p_{\varepsilon}<0<C_{2} p_{\varepsilon}=\beta(0, \varepsilon), \\
& \alpha(1, \varepsilon)=-C_{2} p_{\varepsilon}<0<C_{2} p_{\varepsilon}=\beta(1, \varepsilon) .
\end{aligned}
$$

To be a super solution, $\beta$ must satisfy $F_{\varepsilon} \beta \geq 0$ for $x \in X$. We shall prove this inequality only for $x \in[0,1 / 2]$, since the argument for $x \in[1 / 2,1]$ is similar. In the 
rest of this proof, the notation $\zeta=O(M)$ stands for $|\zeta| \leq \bar{C} M$, where $\bar{C}>0$ is any constant independent of $C_{1}, C_{2}$ and $\varepsilon$.

We set

$$
\hat{w}(\eta, \varepsilon, p)=\left(v_{0}^{0}(\eta, p)+\varepsilon v_{1}^{0}(\eta)\right) \sigma(\varepsilon \eta)
$$

and

$$
\Phi(\eta, \varepsilon, p)=-\frac{\partial^{2} \hat{w}(\eta, \varepsilon, p)}{\partial \eta^{2}}+b\left(\varepsilon \eta, u_{0}(\varepsilon \eta)+\hat{w}(\eta, \varepsilon, p)\right)-p \hat{w}(\eta, \varepsilon, p) ;
$$

these functions are defined for all sufficiently small $|p|$. Then from (2.12),

$$
\Phi(\eta, 0, p)=0 .
$$

This yields

$$
\Phi(\eta, 0,0)=0
$$

and

$$
\left.\frac{\partial \Phi(\eta, \varepsilon, p)}{\partial p}\right|_{(\eta, 0,0)}=0
$$

Next, (2.16) implies that

$$
\left.\frac{\partial \Phi(\eta, \varepsilon, p)}{\partial \varepsilon}\right|_{(\eta, 0,0)}=0 .
$$

By Taylor's theorem and (2.23)-(2.25), we have

$$
\Phi(\eta, \varepsilon, p)=\left.\frac{1}{2}\left(\varepsilon \frac{\partial}{\partial \varepsilon}+p \frac{\partial}{\partial p}\right)^{2} \Phi(\eta, \varepsilon, p)\right|_{(\eta, \theta \varepsilon, \theta p)}
$$

for $\eta>0, \quad 0<\varepsilon \leq 1$ and $0<p \leq p_{0}$ ( $p_{0}$ chosen so that $\hat{w}$ is well defined for $\left.|p|<p_{0}\right)$, and some $\bar{\theta} \in(0,1)$.

Lemmas 2.1-2.3 imply that

$$
0<\frac{\partial \hat{w}(\eta, \varepsilon, p)}{\partial p} \leq C
$$

and

$$
\left|\frac{\partial^{j} \hat{w}(\eta, \varepsilon, p)}{\partial \eta^{j}}\right| \leq C \exp (-(\bar{b}-\delta) \eta)
$$

for $\eta>0$ and $j=0,1, \ldots, J$, where $b_{0}^{2}>p, \bar{b}=\sqrt{b_{0}^{2}-p}\left(b_{0}\right.$ is given by (1.2)) and $\delta$ is any fixed number in $(0, \bar{b})$. Hence there exists a constant $C$ such that

$$
\left|\frac{\partial^{2} \Phi(\eta, \varepsilon, p)}{\partial \varepsilon^{i} \partial p^{2-i}}\right|_{(\eta, \theta \varepsilon, \theta p)} \mid \leq C,
$$

for $i=0,1,2, \quad \eta>0, \quad 0<\varepsilon \leq 1$ and $0<p \leq p_{0}$. Thus (2.26) implies that

$$
|\Phi(\eta, \varepsilon, p)| \leq C\left(\varepsilon^{2}+p^{2}\right),
$$

for $\eta>0, \quad 0<\varepsilon \leq 1$ and $0<p \leq p_{0}$. 
We are now ready to show that $\beta(x, \varepsilon)$ is a super solution of problem (B). For, observing that $w(x, \varepsilon, p)=\hat{w}(\eta, \varepsilon, p)$ when $\eta=x / \varepsilon$, we have

$$
\begin{aligned}
F_{\varepsilon} \beta(x, \varepsilon) & =-\varepsilon^{2} \beta^{\prime \prime}(x, \varepsilon)+b(x, \beta) \\
= & -\varepsilon^{2} u_{0}^{\prime \prime}(x)+\Phi\left(\eta, \varepsilon, C_{1} p_{\varepsilon}\right)+C_{1} p_{\varepsilon} \hat{w}\left(\eta, \varepsilon, C_{1} p_{\varepsilon}\right) \\
& +b\left(x, u_{0}(x)+\hat{w}\left(\eta, \varepsilon, C_{1} p_{\varepsilon}\right)+C_{2} p_{\varepsilon}\right) \\
& -b\left(x, u_{0}(x)+\hat{w}\left(\eta, \varepsilon, C_{1} p_{\varepsilon}\right)\right) \\
= & C_{1} p_{\varepsilon} \hat{w}\left(\eta, \varepsilon, C_{1} p_{\varepsilon}\right)+C_{2} p_{\varepsilon} b_{u}\left(x, u_{0}(x)+\hat{w}\left(\eta, \varepsilon, C_{1} p_{\varepsilon}\right)+\hat{\theta} C_{2} p_{\varepsilon}\right) \\
& +O\left(\varepsilon^{2}+p_{\varepsilon}^{2}\right),
\end{aligned}
$$

by $(2.29)$, for some $\hat{\theta} \in(0,1)$.

Now (1.4a) states that $b_{u}\left(x, u_{0}(x)\right) \geq b_{0}^{2}>0$, so by the compactness of $X$ there exists a constant $C_{3}>0$ such that

$$
b_{u}\left(x, u_{0}(x)+g(x)\right) \geq b_{0}^{2} / 2 \text { for } x \in X,
$$

for all functions $g$ satisfying $|g(x)| \leq C_{3}$ on $X$.

Choose $C_{2}=C_{3} / 2$, so that in $(2.30)$ we get $\left|\hat{\theta} C_{2} p_{\varepsilon}\right| \leq C_{3} / 2$. Set

$$
\begin{gathered}
C_{4}=\max \left\{\left|b_{u}\left(x, u_{0}(x)+\hat{w}(\eta, \varepsilon, r)+s\right)\right|: 0 \leq x \leq 1, \quad \eta>0,\right. \\
\left.0<\varepsilon \leq 1, \quad 0 \leq r \leq p_{0}, \quad 0 \leq s \leq C_{3} / 2\right\} ;
\end{gathered}
$$

it follows from (2.27) and (2.28) that this maximum is well defined. Now (2.30) and Lemmas 2.1-2.3 imply that

$$
F_{\varepsilon} \beta(x, \varepsilon) \geq \begin{cases}p_{\varepsilon}\left(C_{2} b_{0}^{2} / 2-\varepsilon C_{1}\left\|v_{1}^{0}\right\|_{\infty}\right)+O\left(\varepsilon^{2}+p_{\varepsilon}^{2}\right) & \text { if } \hat{w} \leq C_{3} / 2, \\ \left(C_{1}-C_{4}\right) C_{3} p_{\varepsilon} / 2+O\left(\varepsilon^{2}+p_{\varepsilon}^{2}\right) & \text { if } \hat{w}>C_{3} / 2,\end{cases}
$$

where $\left\|v_{1}^{0}\right\|_{\infty}=\max _{\eta \geq 0}\left|v_{1}^{0}(\eta)\right|$. Choose $C_{1}=2 C_{4}$. Then for all sufficiently small $\varepsilon$, we have

$$
F_{\varepsilon} \beta(x, \varepsilon)>0 \text { for } x \in[0,1 / 2] .
$$

Analogously, one may show that $F_{\varepsilon} \beta(x, \varepsilon)>0$ for $x \in[1 / 2,1]$, and that $F_{\varepsilon} \alpha(x, \varepsilon)<$ 0 for $x \in X$.

Theorem 2.1. Under the same hypotheses as in Lemma 2.4, problem (B) has a solution $u(x)$, which is the only solution satisfying

$$
\alpha(x, \varepsilon) \leq u(x) \leq \beta(x, \varepsilon) \quad \text { for } x \in X .
$$

Here, $\beta(x, \varepsilon)$ and $\alpha(x, \varepsilon)$ are the super and sub solutions given by (2.20) and (2.21).

Proof. D'Annunzio [2, Corollary 3.1] proves the following extension of Nagumo's theorem: if problem (B) has a super solution $\beta(x, \varepsilon)$ and a sub solution $\alpha(x, \varepsilon)$, then there exists a solution $u(x)$ of problem $(\mathrm{B})$ such that

$$
\alpha(x, \varepsilon) \leq u(x) \leq \beta(x, \varepsilon) \text { for } x \in X .
$$

Hence the existence of a solution is implied by Lemma 2.4 above. The uniqueness of the solution satisfying (2.31) can be shown by arguments similar to those of $[2$, Theorem 3.6], using degree theory.

Recall that $x(x, \varepsilon, p)=\hat{w}(\eta, \varepsilon, p)$ when $\eta=x / \varepsilon$. From (2.27) and the definitions of our super solution $\beta(x, \varepsilon)$ and sub solution $\alpha(x, \varepsilon)$, we see that

$$
|\beta(x, \varepsilon)-\alpha(x, \varepsilon)| \leq C \varepsilon^{2} \ln ^{2}(1 / \varepsilon) \quad \text { for } x \in X .
$$


This shows that we have tighter control on the solution $u(x)$ of Theorem 2.1 than in Corollary 3.4 in D'Annunzio [2], where the super and sub solutions yield only an $O(\varepsilon)$ estimate of $u$.

In principle one could obtain an approximation to $u$ by explicitly computing $\alpha(x)$ or $\beta(x)$. This would entail solving two nonlinear and two linear second-order differential equations (see $(2.12)-(2.19))$ and would be more complicated than using the difference scheme below to solve (1.1) directly.

\section{A Central Difference SCheme On a Shishkin Mesh}

We analyze the truncation error of a central difference scheme applied to problem (B) on a Shishkin mesh.

For a given positive integer $N$, we denote by $X^{N}$ an arbitrary mesh

$$
0=x_{0}<x_{1}<\cdots<x_{N-1}<x_{N}=1,
$$

with $h_{i}=x_{i}-x_{i-1}$, for $i=1, \ldots, N$, and $\bar{h}_{i}=\left(h_{i}+h_{i+1}\right) / 2$, for $i=1, \ldots, N-1$.

We use $\mathcal{R}^{N+1}$ to denote the real $(N+1)$-dimensional linear space of all column vectors

$$
z=\left(z_{0}, z_{1}, \ldots, z_{N}\right)^{T} .
$$

In what follows, for any function $y \in C(X)$, we shall abuse the notation by also writing $y \in \mathcal{R}^{N+1}$ with $y_{i}=y\left(x_{i}\right)$ for $i=0,1, \ldots, N$.

We equip the space $\mathcal{R}^{N+1}$ with the usual $l_{\infty}$-norm:

$$
\|z\|_{\infty}=\max _{0 \leq i \leq N}\left|z_{i}\right| \text {. }
$$

The induced norm of a linear mapping $\bar{A}=\left(a_{i j}\right): \mathcal{R}^{N+1} \rightarrow \mathcal{R}^{N+1}$ is

$$
\|\bar{A}\|_{\infty}=\max _{0 \leq i \leq N} \sum_{j=0}^{N}\left|a_{i j}\right| .
$$

Let

$$
A=\left(\begin{array}{ccccccc}
-\varepsilon^{-2} & 0 & 0 & & & & \\
r_{1}^{-} & r_{1}^{c} & r_{1}^{+} & & & & \\
& \cdot & \cdot & \cdot & & & \\
& & \cdot & \cdot & \cdot & & \\
& & & \cdot & \cdot & \cdot & \\
& & & & r_{N-1}^{-} & r_{N-1}^{c} & r_{N-1}^{+} \\
& & & & 0 & 0 & -\varepsilon^{-2}
\end{array}\right)
$$

be an $(N+1) \times(N+1)$ tridiagonal matrix, where

$$
r_{i}^{-}=\frac{1}{h_{i} \bar{h}_{i}}, \quad r_{i}^{c}=-\frac{2}{h_{i} h_{i+1}}, \quad r_{i}^{+}=\frac{1}{h_{i+1} \bar{h}_{i}} .
$$

Let $B: \mathcal{R}^{N+1} \rightarrow \mathcal{R}^{N+1}$ be the mapping:

$$
(B z)_{i}= \begin{cases}0 & \text { for } i=0 \\ b\left(x_{i}, z_{i}\right) & \text { for } i=1, \ldots, N-1, \\ 0 & \text { for } i=N\end{cases}
$$

Set

$$
F=-\varepsilon^{2} A+B
$$


We shall use $\left\{F, X^{N}\right\}$ to denote the three-point central difference scheme

$$
F u^{N}=0 .
$$

Let $y \in C^{2}(X)$. Define $\left(F_{\varepsilon} y\right)(0)=y(0)$ and $\left(F_{\varepsilon} y\right)(1)=y(1)$. The truncation error of $F$ in approximating $F_{\varepsilon}$ in terms of $y$ is defined to be $\left\|F y-F_{\varepsilon} y\right\|_{\infty}$. It is clear that $(F y)_{0}=\left(F_{\varepsilon} y\right)(0)$ and $(F y)_{N}=\left(F_{\varepsilon} y\right)(1)$. We shall bound $\left|(F y)_{i}-\left(F_{\varepsilon} y\right)\left(x_{i}\right)\right|$, for $i=1,2, \ldots, N-1$, in the truncation error analysis of this section.

Since $u^{\prime}(x)$ is in general unbounded in the boundary layers at $x=0$ and $x=1$ when $\varepsilon \rightarrow 0$, a polynomial-based discretization cannot be consistent, uniformly in $\varepsilon$, unless it is constructed on a special mesh. In the literature, several types of special graded meshes have been introduced for singularly perturbed two-point boundary value problems; see Herceg [7], D'Annunzio [2] and Gartland [5]. In the present paper we shall use a Shishkin mesh [15], which is piecewise equidistant and consequently much simpler than the above meshes.

Given a positive integer $N$, where $N$ is divisible by 4 , we divide the interval $[0,1]$ into the three subintervals

$$
[0, \sigma], \quad[\sigma, 1-\sigma], \quad \text { and } \quad[1-\sigma, 1]
$$

We use equidistant meshes on each of these subintervals, with $1+N / 4$ points in each of $[0, \sigma]$ and $[1-\sigma, 1]$, and $1+N / 2$ points in $[\sigma, 1-\sigma]$. Set $\tilde{b}_{0}=\min \left\{b_{0}, 1\right\}$. We define the parameter $\sigma$ by

$$
\sigma=\min \left\{1 / 4, \quad 4 \tilde{b}_{0}^{-1} \varepsilon \ln N\right\},
$$

which depends on $\varepsilon$ and $N$. The basic idea here is to use a fine mesh to resolve part of the boundary layers.

More explicitly, we define

$$
X_{s}^{N}: 0=x_{0}<x_{1}<\cdots<x_{i_{0}}<\cdots<x_{N-i_{0}}<\cdots<x_{N}=1,
$$

with $i_{0}=N / 4, x_{i_{0}}=\sigma, x_{N-i_{0}}=1-\sigma$, and

$$
\begin{gathered}
h_{i}=4 \sigma N^{-1} \quad \text { for } i=1, \ldots, i_{0}, N-i_{0}+1, \ldots, N, \\
h_{i}=2(1-2 \sigma) N^{-1} \quad \text { for } i=i_{0}+1, \ldots, N-i_{0} .
\end{gathered}
$$

If $\sigma=1 / 4$, i.e., $1 / 4 \leq 4 \tilde{b}_{0}^{-1} \varepsilon \ln N$, then $N^{-1}$ is very small relative to $\varepsilon$. This is unlikely in practice (and in this case the method can be analyzed using standard techniques). We therefore assume that

$$
\sigma=4 \tilde{b}_{0}^{-1} \varepsilon \ln N .
$$

From (3.3) and (3.4), it is clear that the interval lengths satisfy

$$
h_{i}=16 \tilde{b}_{0}^{-1} \varepsilon N^{-1} \ln N
$$

for $i=1, \ldots, i_{0}, N-i_{0}+1, \ldots, N$, and

$$
N^{-1} \leq h_{i} \leq 2 N^{-1},
$$

for $i=i_{0}+1, \ldots, N-i_{0}$.

Lemma 3.1. Let $y \in C^{4}(X)$. Suppose that $y=Y+V$, where

$$
\left|Y^{(j)}(x)\right| \leq C
$$


and

$$
\left|V^{(j)}(x)\right| \leq C \varepsilon^{-j}\left[\exp \left(-\tilde{b}_{0} x / 2 \varepsilon\right)+\exp \left(-\tilde{b}_{0}(1-x) / 2 \varepsilon\right)\right],
$$

for $x \in X$ and $j=0, \ldots, 4$. Then on the Shishkin mesh $X_{s}^{N}$, the truncation error of the scheme (3.1) satisfies

$$
\left\|F y-F_{\varepsilon} y\right\|_{\infty} \leq C\left(\varepsilon^{2} N^{-1}+N^{-2} \ln ^{2} N\right) .
$$

Proof. Suppose first that $x_{i}$ is inside the fine mesh, i.e., that

$$
i \in\left\{1, \ldots, i_{0}-1\right\} \cup\left\{N-i_{0}+1, \ldots, N-1\right\} .
$$

By a Taylor expansion, there exist $\xi_{i} \in\left(x_{i-1}, x_{i}\right)$ and $\eta_{i} \in\left(x_{i}, x_{i+1}\right)$ such that

$$
\begin{aligned}
(F y)_{i} & -\left(F_{\varepsilon} y\right)\left(x_{i}\right) \\
& =\frac{h_{i}^{2}-h_{i+1}^{2}}{6 \bar{h}_{i}} \varepsilon^{2} y^{\prime \prime \prime}\left(x_{i}\right)-\frac{h_{i}^{3}}{24 \bar{h}_{i}} \varepsilon^{2} y^{(4)}\left(\xi_{i}\right)-\frac{h_{i+1}^{3}}{24 \bar{h}_{i}} \varepsilon^{2} y^{(4)}\left(\eta_{i}\right) \\
& =-\frac{h_{i}^{3}}{24 \bar{h}_{i}} \varepsilon^{2} y^{(4)}\left(\xi_{i}\right)-\frac{h_{i+1}^{3}}{24 \bar{h}_{i}} \varepsilon^{2} y^{(4)}\left(\eta_{i}\right),
\end{aligned}
$$

since the mesh is equidistant on $[0, \sigma] \cup[1-\sigma, 1]$. It is then easy to see, from (3.6), (3.8) and (3.9), that

$$
\left|(F y)_{i}-\left(F_{\varepsilon} y\right)\left(x_{i}\right)\right| \leq C N^{-2} \ln ^{2} N .
$$

Now suppose that $x_{i}$ is no longer inside the fine mesh, i.e., suppose that $i \in$ $\left\{i_{0}, \ldots, N-i_{0}\right\}$. From (3.9) we see that as $\varepsilon \rightarrow 0$ with $N$ fixed, $\varepsilon^{2}\left|y^{\prime \prime \prime}(x)\right|$ is unbounded for $x \in J_{\varepsilon} \equiv\left[\sigma, \sigma_{\varepsilon}\right) \cup\left(1-\sigma_{\varepsilon}, 1-\sigma\right]$, where $\sigma_{\varepsilon}=\min \left\{1 / 4,4 \tilde{b}_{0}^{-1} \varepsilon \ln (1 / \varepsilon)\right\}$. Recall that the Shishkin mesh $X_{s}^{N}$ is coarse on $J_{\varepsilon}$. Hence, (3.11) will not yield a bound for $\left|(F y)_{i}-\left(F_{\varepsilon} y\right)\left(x_{i}\right)\right|$ that is uniform in $\varepsilon$.

We therefore use a Taylor expansion with integral remainder to control $V$. The truncation error of the scheme may be split in the form

$$
(F y)_{i}-\left(F_{\varepsilon} y\right)\left(x_{i}\right)=\left(I_{Y}\right)_{i}+\left(I_{V}\right)_{i} .
$$

Here (see (3.11)),

$$
\left(I_{Y}\right)_{i}=\frac{h_{i}^{2}-h_{i+1}^{2}}{6 \bar{h}_{i}} \varepsilon^{2} Y^{\prime \prime \prime}\left(x_{i}\right)-\frac{h_{i}^{3}}{24 \bar{h}_{i}} \varepsilon^{2} Y^{(4)}\left(\xi_{i}\right)-\frac{h_{i+1}^{3}}{24 \bar{h}_{i}} \varepsilon^{2} Y^{(4)}\left(\eta_{i}\right),
$$

where $\xi_{i} \in\left(x_{i-1}, x_{i}\right)$ and $\eta_{i} \in\left(x_{i}, x_{i+1}\right)$ depend now on the function $Y$, and

$$
\left(I_{V}\right)_{i}=\frac{\varepsilon^{2}}{2 h_{i} \bar{h}_{i}} \int_{x_{i-1}}^{x_{i}}\left(s-x_{i-1}\right)^{2} V^{\prime \prime \prime}(s) d s-\frac{\varepsilon^{2}}{2 h_{i+1} \bar{h}_{i}} \int_{x_{i}}^{x_{i+1}}\left(x_{i+1}-s\right)^{2} V^{\prime \prime \prime}(s) d s .
$$

Then we easily get

$$
\left|\left(I_{Y}\right)_{i}\right| \leq C \varepsilon^{2} N^{-1}
$$


by (3.7) and (3.8). As for $\left|\left(I_{V}\right)_{i}\right|,(3.14)$ and (3.9) give

$$
\begin{aligned}
\left|\left(I_{V}\right)_{i}\right| & \leq C \varepsilon^{-1} \int_{x_{i-1}}^{x_{i+1}}\left(\exp \left(-\tilde{b}_{0} s / 2 \varepsilon\right)+\exp \left(-\tilde{b}_{0}(1-s) / 2 \varepsilon\right)\right) d s \\
& \leq C \varepsilon^{-1} \int_{x_{i_{0}-1}}^{x_{N-i_{0}+1}}\left(\exp \left(-\tilde{b}_{0} s / 2 \varepsilon\right)+\exp \left(-\tilde{b}_{0}(1-s) / 2 \varepsilon\right)\right) d s \\
& =\frac{2 C}{\tilde{b}_{0}}\left(\exp \left(-\tilde{b}_{0} x_{i_{0}-1} / 2 \varepsilon\right)-\exp \left(-\tilde{b}_{0} x_{N-i_{0}+1} / 2 \varepsilon\right)\right) \\
& \leq C \exp \left(-\tilde{b}_{0} x_{i_{0}-1} / 2 \varepsilon\right) \\
& =C N^{-2} \exp \left(\tilde{b}_{0} h_{i_{0}} / 2 \varepsilon\right), \text { since } x_{i_{0}}=\sigma=4 \varepsilon \tilde{b}_{0}^{-1} \ln N, \\
& \leq C N^{-2},
\end{aligned}
$$

by (3.6).

Thus, from $(3.13)-(3.16)$, we obtain

$$
\left|(F y)_{i}-\left(F_{\varepsilon} y\right)\left(x_{i}\right)\right| \leq C\left(\varepsilon^{2} N^{-1}+N^{-2}\right) \quad \text { for } i \in\left\{i_{0}, \ldots, N-i_{0}\right\} .
$$

Combining this with (3.12) completes the proof.

Under the reasonable assumption $\varepsilon \leq N^{-1}$, the estimate (3.10) becomes $\left\|F y-F_{\varepsilon} y\right\|_{\infty} \leq C N^{-2} \ln ^{2} N$. This is much better than the $O(h)$ result obtained by D'Annunzio [2] for the same scheme with a more complicated mesh, where $h$ is the maximum mesh spacing.

\section{UNIFORM CONVERGENCE}

We use degree theory to investigate the existence and uniform convergence of solutions of the central difference scheme on the Shishkin mesh $X_{s}^{N}$ for problem (B). We shall prove that the method is uniformly convergent of order $N^{-2} \ln ^{2} N$ on this piecewise equidistant mesh.

For this purpose, we imbed problem (B) in the following family of problems:

$$
\begin{aligned}
& \tilde{F}_{\varepsilon}(\tilde{u}, t) \equiv-\varepsilon^{2} \tilde{u}_{x x}(x, t)+\tilde{b}(x, t, \tilde{u}(x, t))=0 \quad \text { for } x \in(0,1), \\
& \tilde{u}(0, t)=\tilde{u}(1, t)=0,
\end{aligned}
$$

where $t \in[0,1]$ is a parameter,

$$
\tilde{b}(x, t, \tilde{u}(x, t))=t b(x, \tilde{u}(x, t))+(1-t)\left(\tilde{u}(x, t)-u_{0}(x)\right),
$$

for $(x, t, \tilde{u}) \in[0,1] \times[0,1] \times \mathcal{R}^{1}$, and $u_{0}$ is the solution of (1.3). Note that for each $x$ and $t$ we have $\tilde{b}\left(x, t, u_{0}(x)\right)=0$.

Recall $\tilde{b}_{0}=\min \left\{b_{0}, 1\right\}$. We have

$$
\begin{aligned}
\tilde{b}_{u}(x, t, u) & =t b_{u}(x, u)+(1-t) \\
& =t b_{0}^{2}+(1-t) \\
& \geq \tilde{b}_{0}^{2}
\end{aligned}
$$

for all $(x, t, u) \in[0,1] \times[0,1] \times \mathcal{R}^{1}$. Hence, for each $t$, problem $(4.1)-(4.2)$ is of the same type as problem (B).

Define the mapping $\tilde{F}(\cdot, \cdot): \mathcal{R}^{N+1} \times[0,1] \rightarrow \mathcal{R}^{N+1}$ by

$$
\tilde{F}(z, t)=-\varepsilon^{2} A z+\tilde{B}(z, t),
$$


where $\tilde{B}(\cdot, \cdot): \mathcal{R}^{N+1} \times[0,1] \rightarrow \mathcal{R}^{N+1}$ is given by

$$
(\tilde{B}(z, t))_{i}= \begin{cases}0 & \text { for } i=0 \\ \tilde{b}\left(x_{i}, t, z_{i}\right) & \text { for } i=1, \ldots, N-1, \\ 0 & \text { for } i=N .\end{cases}
$$

Then the scheme (3.1) for problem (B) is imbedded in the family of schemes

$$
\tilde{F}(z, t)=0 .
$$

Let us introduce some more notation and definitions.

For $z^{1}$ and $z^{2} \in \mathcal{R}^{N+1}$, we denote by $z^{1} \leq z^{2}$ (or $z^{1}<z^{2}$ ) the natural partial ordering on $\mathcal{R}^{N+1}$, i.e., $z_{i}^{1} \leq z_{i}^{2}$ (or $z_{i}^{1}<z_{i}^{2}$ ) for $i=0,1, \ldots, N$.

Let $M: \mathcal{R}^{N+1} \rightarrow \mathcal{R}^{N+1}$ be a mapping. Let $\alpha, \beta \in \mathcal{R}^{N+1}$. If

$$
\begin{aligned}
& M \alpha<0 \\
& M \beta>0
\end{aligned}
$$

and

$$
\alpha<\beta
$$

then $\beta$ and $\alpha$ are said to be super and sub solutions of $M z=0$, respectively.

Let $\alpha, \beta \in \mathcal{R}^{N+1}$ satisfy $\alpha<\beta$. Let $G$ be a mapping: $\mathcal{R}^{N+1} \rightarrow \mathcal{R}^{N+1}$. Define $G^{m}: \mathcal{R}^{N+1} \rightarrow \mathcal{R}^{N+1}$ by

$$
\left(G^{m} z\right)_{i}= \begin{cases}(G \beta)_{i}+\left(z_{i}-\beta_{i}\right) & \text { if } z_{i}>\beta_{i}, \\ (G z)_{i} & \text { if } \alpha_{i} \leq z_{i} \leq \beta_{i}, \\ (G \alpha)_{i}+\left(\alpha_{i}-z_{i}\right) & \text { if } z_{i}<\alpha_{i},\end{cases}
$$

for $i=0,1, \ldots, N$. Then $G^{m}$ is called a modification of $G$.

We give a strengthening of Theorem 5.1 of D'Annunzio [2].

Lemma 4.1. Let $D=\left(d_{i j}\right)$ be an $(N+1) \times(N+1)$ matrix satisfying

$$
d_{i j} \leq 0 \quad \text { for } 0 \leq i, j \leq N, \quad i \neq j
$$

and

$$
\sum_{j=1}^{N} d_{i j} \geq 0 \quad \text { for } 0 \leq i \leq N
$$

Let $G: \mathcal{R}^{N+1} \rightarrow \mathcal{R}^{N+1}$ be a mapping. Let $\alpha, \beta \in \mathcal{R}^{N+1}$ satisfy $\alpha<\beta$. Let $G^{m}$ be as in (4.7). Define $M: \mathcal{R}^{N+1} \rightarrow \mathcal{R}^{N+1}$ by

$$
M=D+G^{m} \text {. }
$$

If

$$
\begin{aligned}
& M z=0, \\
& M \alpha<0
\end{aligned}
$$

and

$$
M \beta>0,
$$

then

$$
\alpha<z<\beta \text {. }
$$


Proof. We shall prove only that $z<\beta$, since $z>\alpha$ may be shown analogously.

Set $\nu=z-\beta$. We prove that $\nu<0$. Suppose that $\nu<0$ is false. Then for some $i \in\{0,1, \ldots, N\}$, we have $\nu_{i} \geq 0$. Let $k$ be an integer such that

$$
\nu_{k}=\max _{0 \leq i \leq N}\left\{\nu_{i}\right\}
$$

Clearly

$$
\nu_{k} \geq 0
$$

By (4.10),

$$
\begin{aligned}
0 & =(D z)_{k}+\left(G^{m} z\right)_{k} \\
& =(D z)_{k}+(G \beta)_{k}+\left(z_{k}-\beta_{k}\right),
\end{aligned}
$$

from (4.7) and (4.14). Hence, using (4.7) and (4.12), we get

$$
\begin{aligned}
-\nu_{k} & =(D z)_{k}+(G \beta)_{k} \\
& =(D z)_{k}+\left(G^{m} \beta\right)_{k} \\
& >(D z)_{k}-(D \beta)_{k} \\
& =(D \nu)_{k} \\
& =\sum_{j=0}^{N} d_{k j} \nu_{j} \\
& \geq\left(\sum_{j=0}^{N} d_{k j}\right) \nu_{k}, \quad \text { by (4.8) and (4.13), } \\
& \geq 0,
\end{aligned}
$$

by (4.9) and (4.14). That is, $\nu_{k}<0$. This contradicts (4.14) and the proof of Lemma 4.1 is completed.

D'Annunzio [2] proved the same result under the extra conditions $d_{i i}>0$ for $i=0,1, \ldots, N$, while assuming that strict inequality holds in (4.9) for at least one $i$.

For each $t \in[0,1]$, set

$$
\tilde{w}(x, t, \varepsilon, p)=\left\{\begin{array}{r}
\left(\tilde{v}_{0}^{0}(x / \varepsilon, t, p)+\varepsilon \tilde{v}_{1}^{0}(x / \varepsilon, t)\right) \sigma(x) \quad \text { for } 0 \leq x \leq 1 / 2 \\
\left(\tilde{v}_{0}^{1}((1-x) / \varepsilon, t, p)+\varepsilon \tilde{v}_{1}^{1}((1-x) / \varepsilon, t)\right) \sigma(1-x) \\
\text { for } 1 / 2<x \leq 1
\end{array}\right.
$$

where $\tilde{v}_{0}^{0}(\eta, t, p), \tilde{v}_{0}^{1}(\eta, t, p), \tilde{v}_{1}^{0}(\eta, t)$, and $\tilde{v}_{1}^{1}(\eta, t)$ are respectively defined by

$$
\begin{aligned}
& \ddot{\tilde{v}}_{0}^{0}-\tilde{b}\left(0, t, u_{0}(0)+\tilde{v}_{0}^{0}\right)=-p \tilde{v}_{0}^{0} \quad \text { for } \eta>0, \\
& \tilde{v}_{0}^{0}(0, t, p)=-u_{0}(0), \quad \tilde{v}_{0}^{0}(\infty, t, p)=0, \\
& \ddot{v}_{0}^{1}-\tilde{b}\left(1, t, u_{0}(1)+\tilde{v}_{0}^{1}\right)=-p \tilde{v}_{0}^{1} \quad \text { for } \eta>0, \\
& \tilde{v}_{0}^{1}(0, t, p)=-u_{0}(1), \quad \tilde{v}_{0}^{1}(\infty, t, p)=0
\end{aligned}
$$




$$
\begin{aligned}
& \ddot{\tilde{v}}_{1}^{0}-\tilde{b}_{u}\left(0, t, u_{0}(0)+\tilde{v}_{0}^{0}(\eta, 0)\right) \tilde{v}_{1}^{0} \\
& =\eta\left[\tilde{b}_{x}\left(0, t, u_{0}(0)+\tilde{v}_{0}^{0}(\eta, 0)\right)+\tilde{b}_{u}\left(0, t, u_{0}(0)+\tilde{v}_{0}^{0}(\eta, 0)\right) u_{0}^{\prime}(0)\right] \\
& \quad \text { for } \eta>0 \\
& \tilde{v}_{1}^{0}(0, t)=0, \quad \tilde{v}_{1}^{0}(\infty, t)=0,
\end{aligned}
$$

and

$$
\begin{aligned}
& \ddot{\tilde{v}}_{1}^{1}-\tilde{b}_{u}\left(1, t, u_{0}(1)+\tilde{v}_{0}^{1}(\eta, 0)\right) \tilde{v}_{1}^{1} \\
& =\eta\left[\tilde{b}_{x}\left(1, t, u_{0}(1)+\tilde{v}_{0}^{1}(\eta, 0)\right)+\tilde{b}_{u}\left(1, t, u_{0}(1)+\tilde{v}_{0}^{1}(\eta, 0)\right) u_{0}^{\prime}(1)\right] \\
& \quad \text { for } \eta>0 \\
& \tilde{v}_{1}^{1}(0, t)=0, \quad \tilde{v}_{1}^{1}(\infty, t)=0 .
\end{aligned}
$$

Recall that $\tilde{b}_{0}$ is independent of $t$ in (4.4). One may show, by the arguments of $\S 2$, that there is a $\tilde{p}_{0}>0$, independent of $\varepsilon$ and $t$, such that $w(x, t, \varepsilon, p)$ is well defined for $|p| \leq \tilde{p}_{0}$. Furthermore, we have

$$
0 \leq \frac{\partial \tilde{w}}{\partial p}(x, t, \varepsilon, p) \leq C
$$

and

$$
\left|\frac{\partial^{j} \tilde{w}}{\partial x^{j}}(x, t, \varepsilon, p)\right| \leq C \varepsilon^{-j}(\exp (-(\tilde{b}-\delta) x / \varepsilon)+\exp (-(\tilde{b}-\delta)(1-x) / \varepsilon))
$$

for $(x, t) \in[0,1] \times[0,1]$ and $j=0,1,2,3,4$. Here, $\tilde{b}_{0}^{2}>p$ and $\tilde{b}=\sqrt{\tilde{b}_{0}^{2}-p}$ with $\tilde{b}_{0}$ given by (4.4) and $\delta$ any fixed number in $(0, \tilde{b})$.

Note that problem $(4.1)-(4.3)$ becomes (1.1) when $t=1$. We have

$$
\tilde{w}(x, 1, \varepsilon, p)=w(x, \varepsilon, p) \quad \text { for } x \in[0,1] .
$$

Assumption 4.1. In what follows, we shall assume that $\varepsilon \leq N^{-1}$, which is nonrestrictive in practice.

Lemma 4.2. Set $\tilde{p}_{N}=N^{-2} \ln ^{2} N$. Let $t \in[0,1]$. Then we can choose positive constants $\tilde{C}_{1}>0$ and $\tilde{C}_{2}>0$, which are independent of $N, \varepsilon$ and $t$, and a positive integer $N_{0}$, which depends on $\tilde{C}_{1}$ and $\tilde{C}_{2}$ but is independent of $\varepsilon$ and $t$, such that for each fixed $t \in[0,1]$, when $N \geq N_{0}$, the functions $\tilde{w}\left(x, t, \varepsilon, \tilde{C}_{1} \tilde{p}_{N}\right)$ and $\tilde{w}\left(x, t, \varepsilon,-\tilde{C}_{1} \tilde{p}_{N}\right)$ are well defined, and

$$
\tilde{\beta}^{N}(x, t)=u_{0}(x)+\tilde{w}\left(x, t, \varepsilon, \tilde{C}_{1} \tilde{p}_{N}\right)+\tilde{C}_{2} \tilde{p}_{N}
$$

and

$$
\tilde{\alpha}^{N}(x, t)=u_{0}(x)+\tilde{w}\left(x, t, \varepsilon,-\tilde{C}_{1} \tilde{p}_{N}\right)-\tilde{C}_{2} \tilde{p}_{N}
$$

are super and sub solutions, respectively, of (4.6) on the Shishkin mesh $X_{4}^{N}$.

Proof. Let

$$
\tilde{\beta}^{N}(x, t)=u_{0}(x)+\tilde{w}\left(x, t, \varepsilon, \tilde{C}_{1} \tilde{p}_{N}\right)+\tilde{C}_{2} \tilde{p}_{N}
$$

and

$$
\tilde{\alpha}^{N}(x, t)=u_{0}(x)+\tilde{w}\left(x, t, \varepsilon,-\tilde{C}_{1} \tilde{p}_{N}\right)-\tilde{C}_{2} \tilde{p}_{N},
$$

where $\tilde{C}_{1}>0$ and $\tilde{C}_{2}>0$ will be chosen later. 
For each $t \in[0,1]$, it is clear that

$$
\tilde{\alpha}^{N}(\cdot, t)<\tilde{\beta}^{N}(\cdot, t)
$$

We now prove that $\tilde{F}\left(\tilde{\beta}^{N}, t\right)>0$. From the definitions of the terms involved,

$$
\left(\tilde{F}\left(\tilde{\beta}^{N}, t\right)\right)_{0}=\left(\tilde{F}\left(\tilde{\beta}^{N}, t\right)\right)_{N}=\tilde{C}_{1} \tilde{p}_{N}>0 .
$$

Fix $i \in\{1,2, \ldots N-1\}$ and $t \in[0,1]$. We have

$$
\begin{aligned}
\left(\tilde{F}\left(\tilde{\beta}^{N}, t\right)\right)_{i}= & \left(\left(\tilde{F}\left(\tilde{\beta}^{N}, t\right)\right)_{i}-\left(\tilde{F}_{\varepsilon}\left(\tilde{\beta}^{N}, t\right)\right)\left(x_{i}, t, \varepsilon\right)\right) \\
& +\left(\tilde{F}_{\varepsilon}\left(\tilde{\beta}^{N}, t\right)\right)\left(x_{i}, t, \varepsilon\right) .
\end{aligned}
$$

We separately analyze these two terms.

First, take $N_{1}>0$ such that $\tilde{C}_{1} \tilde{p}_{N}<\tilde{b}_{0}^{2} / 4$ for $N \geq N_{1}$. Then for $N \geq N_{1}$ and $\delta=\tilde{b}_{0} / 4$ in (4.16), we have

$$
\left|\frac{\partial^{j} \tilde{w}}{\partial x^{j}}\left(x, t, \varepsilon, \tilde{C}_{1} \tilde{p}_{N}\right)\right| \leq \tilde{C} \varepsilon^{-j}\left(\exp \left(-\tilde{b}_{0} x / 2 \varepsilon\right)+\exp \left(-\tilde{b}_{0}(1-x) / 2 \varepsilon\right)\right),
$$

for $(x, t) \in[0,1] \times[0,1]$ and $j=0, \ldots, 4$. On the Shishkin mesh $X_{4}^{N}$ one has, using Lemma 3.1 and $\varepsilon \leq N^{-1}$,

$$
\left|\left(\tilde{F}\left(\tilde{\beta}^{N}, t\right)\right)_{i}-\left(\tilde{F}_{\varepsilon}\left(\tilde{\beta}^{N}, t\right)\right)\left(x_{i}, t, \varepsilon\right)\right| \leq \tilde{C} \tilde{p}_{N},
$$

for some positive constant $\tilde{C}$ that is independent of $\tilde{C}_{1}, \tilde{C}_{2}, N, \varepsilon$ and $t$.

Next, we can easily adapt the proof of Lemma 2.4 to show that

$$
\tilde{F}_{\varepsilon}\left(\tilde{\beta}^{N}, t\right)\left(x_{i}, t, \varepsilon\right) \geq 2 \tilde{C} \tilde{p}_{N},
$$

for sufficiently large $\tilde{C}_{1}$ and $\tilde{C}_{2}$. Hence,

$$
\left(\tilde{F}\left(\tilde{\beta}^{N}, t\right)\right)_{i}>0 \text { for } i=1, \ldots, N-1 .
$$

Combining this with $(4.20)$ yields $\tilde{F}\left(\tilde{\beta}^{N}, t\right)>0$.

We can similarly show that $\tilde{F}\left(\tilde{\alpha}^{N}, t\right)>0$, to complete the proof.

We now introduce a modified problem corresponding to (4.6). Consider

$$
\tilde{F}^{m}(z, t)=0
$$

where the mapping $\tilde{F}^{m}(\cdot, \cdot): \mathcal{R}^{N+1} \times[0,1] \rightarrow \mathcal{R}^{N+1}$ is defined by

$$
\tilde{F}^{m}(z, t)=-\varepsilon^{2} A z+\tilde{B}^{m}(z, t) .
$$

Here, $\tilde{B}^{m}(\cdot, t)$ is the modification of $\tilde{B}(\cdot, t)$, with $\tilde{\beta}^{N}$ and $\tilde{\alpha}^{N}$ given by (4.18) and (4.19), respectively, for each $t$; see (4.7).

Define an open and bounded set $D_{t} \subset \mathcal{R}^{N+1}$ for each $t \in[0,1]$ by

$$
D_{t}=\left\{z \in \mathcal{R}^{N+1}: \tilde{\alpha}^{N}(\cdot, t)<z<\tilde{\beta}^{N}(\cdot, t)\right\} .
$$

We shall denote by $\bar{D}_{t}$ and $\partial D_{t}$ the closure and the boundary, respectively, of $D_{t}$ in $\mathcal{R}^{N+1}$. 
Define the mapping $T(\cdot, \cdot): \bar{D}_{1} \times[0,1] \rightarrow \mathcal{R}^{N+1}$ by

$$
\begin{aligned}
(T(z, t))_{i}= & \left(z_{i}-\tilde{\alpha}^{N}\left(x_{i}, 1\right)\right) \frac{\tilde{\beta}^{N}\left(x_{i}, t\right)}{\tilde{\beta}^{N}\left(x_{i}, 1\right)-\tilde{\alpha}^{N}\left(x_{i}, 1\right)} \\
& +\left(\tilde{\beta}^{N}\left(x_{i}, 1\right)-z_{i}\right) \frac{\tilde{\alpha}^{N}\left(x_{i}, t\right)}{\tilde{\beta}^{N}\left(x_{i}, 1\right)-\tilde{\alpha}^{N}\left(x_{i}, 1\right)},
\end{aligned}
$$

for $i=0,1, \ldots, N$. It is easy to see that, for each $t \in[0,1], T(\cdot, t)$ is a linear transformation from $\bar{D}_{1}$ onto $\bar{D}_{t}$.

We finally define a mapping $\tilde{H}(\cdot, \cdot): \bar{D}_{1} \times[0,1] \rightarrow \mathcal{R}^{N+1}$ by

$$
\tilde{H}(z, t)=\tilde{F}^{m}(T(z, t), t) \quad \text { for }(z, t) \in \bar{D}_{1} \times[0,1] .
$$

This is a continuously differentiable mapping and satisfies

$$
\tilde{H}(z, 1)=\tilde{F}^{m}(z, 1)=\tilde{F}(z, 1)=F z,
$$

for $z \in \bar{D}_{1}$. We shall prove that

$$
\left|\operatorname{Deg}\left(\tilde{H}(\cdot, 1), D_{1}, 0\right)\right|=1
$$

where Deg denotes topological degree (see, e.g., Ortega and Rheinboldt [13]), by using the Homotopy Invariance Theorem [13, Theorem 6.2.2].

We first show the following:

Lemma 4.3. There holds

$$
\tilde{H}(z, t) \neq 0 \quad \text { for all }(z, t) \in \partial D_{1} \times[0,1] .
$$

Proof. Suppose that $\tilde{H}\left(z^{*}, t^{*}\right)=0$ for some $\left(z^{*}, t^{*}\right) \in \bar{D}_{1} \times[0,1]$. Set $T^{*}=T\left(z^{*}, t^{*}\right)$. Then $T^{*} \in \bar{D}_{t^{*}}$ satisfies

$$
\tilde{F}^{m}\left(T^{*}, t^{*}\right)=0 \text {. }
$$

From the definition of $\tilde{F}^{m}(\cdot, \cdot)$ and Lemma 4.2 , we have

$$
\begin{gathered}
\tilde{F}^{m}\left(\tilde{\alpha}^{N}\left(\cdot, t^{*}\right), t^{*}\right)=\tilde{F}\left(\tilde{\alpha}^{N}\left(\cdot, t^{*}\right), t^{*}\right)<0, \\
\tilde{F}^{m}\left(\tilde{\beta}^{N}\left(\cdot, t^{*}\right), t^{*}\right)=\tilde{F}\left(\tilde{\beta}^{N}\left(\cdot, t^{*}\right), t^{*}\right)>0 .
\end{gathered}
$$

If we set $D=-\varepsilon^{2} A$, the conditions (4.8) and (4.9) are satisfied. Combining (4.22) - (4.24) with Lemma 4.1 yields

$$
\tilde{\alpha}^{N}\left(\cdot, t^{*}\right)<T^{*}<\tilde{\beta}^{N}\left(\cdot, t^{*}\right) .
$$

From the definition of $T(\cdot, \cdot)$, we obtain

$$
\begin{aligned}
z_{i}^{*}= & \left(T_{i}^{*}-\tilde{\alpha}^{N}\left(x_{i}, t^{*}\right)\right) \frac{\tilde{\beta}^{N}\left(x_{i}, 1\right)}{\tilde{\beta}^{N}\left(x_{i}, t^{*}\right)-\tilde{\alpha}^{N}\left(x_{i}, t^{*}\right)} \\
& +\left(\tilde{\beta}^{N}\left(x_{i}, t^{*}\right)-T_{i}^{*}\right) \frac{\tilde{\alpha}^{N}\left(x_{i}, 1\right)}{\tilde{\beta}^{N}\left(x_{i}, t^{*}\right)-\tilde{\alpha}^{N}\left(x_{i}, t^{*}\right)},
\end{aligned}
$$

for $i=0,1, \ldots, N$. Hence,

$$
\tilde{\alpha}^{N}(\cdot, 1)<z^{*}<\tilde{\beta}^{N}(\cdot, 1)
$$

i.e., $z^{*} \notin \partial D_{1}$, which is the desired result.

Now we have 
Lemma 4.4. If $\tilde{C}_{1}$ and $\tilde{C}_{2}$ in (4.18) and (4.19) are chosen sufficiently large, then

$$
\left|\operatorname{Deg}\left(\tilde{H}(\cdot, 0), D_{1}, 0\right)\right|=1 \text {. }
$$

Proof. We start with the problem

$$
\tilde{F}(z, 0)=0 \quad \text { for } z \in \mathcal{R}^{N+1} .
$$

Set

$$
S=\left(\begin{array}{ccccccc}
0 & 0 & 0 & & & & \\
0 & 1 & 0 & & & & \\
& \cdot & \cdot & . & & & \\
& & . & . & . & & \\
& & & \cdot & . & . & \\
& & & & 0 & 1 & 0 \\
& & & & 0 & 0 & 0
\end{array}\right) .
$$

Then (4.25) can be written in the form

$$
\left(-\varepsilon^{2} A+S\right) z-S u_{0}=0,
$$

from (4.3) and (4.5). The matrix $-\varepsilon^{2} A+S$ is an M-matrix and thus has a positive inverse. Consequently, (4.25) has a unique solution

$$
z^{*}=\left(-\varepsilon^{2} A+S\right)^{-1} S u_{0} \in \mathcal{R}^{N+1} .
$$

We wish to prove that $z^{*} \in D_{0}$. We have

$$
\begin{aligned}
\left(-\varepsilon^{2} A+S\right)\left(\tilde{\beta}^{N}(\cdot, 0)-z^{*}\right) & =\tilde{F}\left(\tilde{\beta}^{N}(\cdot, 0), 0\right)-\tilde{F}\left(z^{*}, 0\right) \\
& =\tilde{F}\left(\tilde{\beta}^{N}(\cdot, 0), 0\right) \\
& >0
\end{aligned}
$$

by (4.25) and (4.24). Hence,

$$
\tilde{\beta}^{N}(\cdot, 0)>z^{*}
$$

Similarly,

$$
\tilde{\alpha}^{N}(\cdot, 0)<z^{*}
$$

That is, $z^{*} \in D_{0}$.

We now consider the problem

$$
\tilde{H}(z, 0)=0 \quad \text { for } z \in \bar{D}_{1} .
$$

As $T(z, 0) \in \bar{D}_{0}$, the problem $(4.26)$ is equivalent to $\tilde{F}(T(z, 0), 0)=0$. But from above, (4.25) has a unique solution $z^{*} \in D_{0}$. Consequently, we need look only for solutions $z \in \bar{D}_{1}$ of

$$
T(z, 0)=z^{*}
$$

Recalling that $T(\cdot, 0)$ is a linear mapping from $\bar{D}_{1}$ onto $\bar{D}_{0}$, so $\partial D_{0}=T\left(\partial D_{1}, 0\right)$, we conclude that (4.27) has a unique solution $\tilde{z} \in D_{1}$. That is, (4.26) has a unique solution, which lies in $D_{1}$. 
Furthermore, we have for $z \in D_{1}$,

$$
\begin{aligned}
\frac{\partial \tilde{H}}{\partial z}(z, 0) & =\frac{\partial \tilde{F}^{m}}{\partial T}(T(z, 0), 0) \frac{\partial T}{\partial z}(z, 0) \\
& =\frac{\partial \tilde{F}}{\partial T}(T(z, 0), 0) \frac{\partial T}{\partial z}(z, 0) \\
& =\left(-\varepsilon^{2} A+S\right) \frac{\partial T}{\partial z}(z, 0) .
\end{aligned}
$$

From above, we know that

$$
\operatorname{det}\left(-\varepsilon^{2} A+S\right) \neq 0 .
$$

Since $\tilde{\alpha}^{N}(\cdot, 0)<\tilde{\beta}^{N}(\cdot, 0)$, we have

$$
\operatorname{det}\left(\frac{\partial T}{\partial z}(z, 0)\right) \neq 0 \quad \text { for all } z \in \mathcal{R}^{N+1} .
$$

Therefore,

$$
\operatorname{det}\left(\frac{\partial \tilde{H}}{\partial z}(z, 0)\right) \neq 0 \quad \text { for all } z \in D_{1} .
$$

We have shown that (4.26) has a unique solution $z^{*}$, which lies in $D_{1}$, with $\operatorname{det}\left(\left.\frac{\partial \tilde{H}}{\partial z}\right|_{z=z^{*}}\right) \neq 0$. This completes the proof.

Theorem 4.1. Let $u(x)$ be the solution of problem (B) guaranteed by Theorem 2.1. Assume that $\varepsilon \leq N^{-1}$. For $N$ sufficiently large, independently of $\varepsilon$, the scheme $\left\{F, X_{s}^{N}\right\}$ has a solution $u^{N}$ such that

$$
\left\|u-u^{N}\right\|_{\infty} \leq C N^{-2} \ln ^{2} N
$$

Proof. Let $\alpha$ and $\beta$ be given by (2.19) and (2.20). Then

$$
\alpha \leq u \leq \beta,
$$

by Theorem 2.1 .

On the other hand, from Lemma 4.3,

$$
\operatorname{Deg}\left(\tilde{H}(\cdot, t), D_{1}, 0\right) \text { is constant for } t \in[0,1],
$$

by the Homotopy Invariance Theorem [13, Theorem 6.2.2]. Hence,

$$
\left|\operatorname{Deg}\left(\tilde{H}(\cdot, 1), D_{1}, 0\right)\right|=\left|\operatorname{Deg}\left(\tilde{H}(\cdot, 0), D_{1}, 0\right)\right|=1,
$$

by Lemma 4.4. This implies that the equation

$$
\tilde{H}(z, 1)=0
$$

has at least one solution $u^{N} \in D_{1}$. Recall (4.21). We see that $\left\{F, X_{s}^{N}\right\}$ has a solution that satisfies

$$
\tilde{\alpha}^{N}(\cdot, 1)<u^{N}<\tilde{\beta}^{N}(\cdot, 1) .
$$

Choose $\tilde{C}_{1}$ and $\tilde{C}_{2}$ in Lemma 4.2 sufficiently large such that $\tilde{C}_{1} \geq C_{1}$ and $\tilde{C}_{2} \geq C_{2}$, where $C_{1}$ and $C_{2}$ are given in Lemma 2.4. Then $C_{1} p_{\varepsilon} \leq \tilde{C}_{1} \tilde{p}_{N}$ and 
$C_{2} p_{\varepsilon} \leq \tilde{C}_{2} \tilde{p}_{N}$, since $\varepsilon \leq N^{-1}$. Hence, for $i=0,1, \ldots, N$,

$$
\begin{aligned}
\beta\left(x_{i}, \varepsilon\right) & =u_{0}\left(x_{i}\right)+w\left(x_{i}, \varepsilon, C_{1} p_{\varepsilon}\right)+C_{2} p_{\varepsilon} \\
& =u_{0}\left(x_{i}\right)+\tilde{w}\left(x_{i}, 1, \varepsilon, C_{1} p_{\varepsilon}\right)+C_{2} p_{\varepsilon} \\
& \leq u_{0}\left(x_{i}\right)+\tilde{w}\left(x_{i}, 1, \varepsilon, \tilde{C}_{1} \tilde{p}_{N}\right)+\tilde{C}_{2} \tilde{p}_{N} \\
& =\tilde{\beta}^{N}\left(x_{i}, 1\right),
\end{aligned}
$$

by (4.17) and (4.15). Similarly, for $i=0,1, \ldots, N$,

$$
\tilde{\alpha}^{N}\left(x_{i}, 1\right) \leq \alpha\left(x_{i}, \varepsilon\right) .
$$

That is,

$$
\tilde{\alpha}^{N}(\cdot, 1) \leq \alpha<\beta \leq \tilde{\beta}^{N}(\cdot, 1) .
$$

We have

$$
\begin{aligned}
\tilde{\beta}^{N}\left(x_{0}, 1\right)-\tilde{\alpha}^{N}\left(x_{0}, 1\right) & =\tilde{\beta}^{N}\left(x_{N}, 1\right)-\tilde{\alpha}^{N}\left(x_{N}, 1\right) \\
& =2 \tilde{C}_{2} N^{-2} \ln ^{2} N
\end{aligned}
$$

and, for $i=1, \ldots, N-1$,

$$
\begin{aligned}
& \left|\tilde{\beta}^{N}\left(x_{i}, 1\right)-\tilde{\alpha}^{N}\left(x_{i}, 1\right)\right| \\
& \leq 2 \tilde{C}_{1} N^{-2} \ln ^{2} N\left|\frac{\partial w}{\partial p}\left(x_{i}, \varepsilon, p^{*}\right)\right|+2 \tilde{C}_{2} N^{-2} \ln ^{2} N, \\
& \quad \text { where } p^{*} \in\left(-\tilde{C}_{1} \tilde{p}_{N}, \tilde{C}_{1} \tilde{p}_{N}\right), \\
& \leq C N^{-2} \ln ^{2} N,
\end{aligned}
$$

by $(2.27)$ and the relation $w(x, \varepsilon, p)=\hat{w}(x / \varepsilon, \varepsilon, p)$.

Therefore, from (4.28) and (4.29) - (4.32),

$$
\left\|u-u^{N}\right\|_{\infty} \leq\left\|\tilde{\beta}^{N}(\cdot, 1)-\tilde{\alpha}^{N}(\cdot, 1)\right\|_{\infty} \leq C N^{-2} \ln ^{2} N,
$$

which is the desired result.

Theorem 4.1 achieves uniform accuracy that is almost one order higher than that of D'Annunzio [2], who uses a more complicated locally quasi-equidistant mesh.

\section{Numerical Results}

In this section we present numerical results to confirm the uniform accuracy of the scheme $\left\{F, X_{s}^{N}\right\}$.

The nonlinear system of equations is solved using Newton's method with the initial guess $u^{N, 0}=\left(0, u_{0}\left(x_{1}\right), \ldots, u_{0}\left(x_{N-1}\right), 0\right)^{T}$. Here, $u_{0}$ is a stable reduced solution with stable boundary layers. We iteratively compute $u^{N, k}$, for $k=1,2, \ldots$, as successive approximations to $u^{N}$. The stopping criterion used is

$$
\max \left\{\left\|F u^{N, k}\right\|_{\infty},\left\|u^{N, k}-u^{N, k-1}\right\|_{\infty}\right\}<0.1 N^{-2} .
$$

For each $N$ and $\varepsilon$ in the tables, it takes only about five iterations to satisfy this criterion.

The exact solutions of our test problems are unknown. We use a double-mesh method [3] to compute the experimental rates of convergence. In order to do this, we shall in addition to computing $u^{N}$ also compute another approximate solution $\tilde{u}^{N}$ that we now describe. 
Let $\tilde{u}^{N} \in \mathcal{R}^{N+1}$ be a solution of $\left\{F, \tilde{X}_{s}^{N}\right\}$, where $\tilde{X}_{s}^{N}$ is a Shishkin mesh with the mesh parameter $\sigma$ of (3.2) altered slightly to

$$
\tilde{\sigma}=\min \left\{1 / 4,4 \tilde{b}_{0}^{-1} \varepsilon \ln (N / 2)\right\} .
$$

Then for $i=0,1, \ldots, N$, the $i$ th point of the mesh $X_{s}^{N}$ coincides with the $(2 i)$ th point of the mesh $\tilde{X}_{s}^{2 N}$.

By inspecting the arguments of $\S \S 3$ and 4 , one may see that when $\varepsilon \leq N^{-1}$,

$$
\left\|u-\tilde{u}^{N}\right\|_{\infty} \leq C\left(N^{-1} \ln N\right)^{2},
$$

where $C$ is independent of $N$ and $\varepsilon$. Hence, for $i=0,1, \ldots, N$,

$$
\left|\left(u^{N}\right)_{i}-\left(\tilde{u}^{2 N}\right)_{2 i}\right| \leq C\left(N^{-1} \ln N\right)^{2} .
$$

For each $N$ and $\varepsilon$, we shall report

$$
\tilde{E}_{\varepsilon}^{N}=\max _{0 \leq i \leq N}\left|\left(u^{N}\right)_{i}-\left(\tilde{u}^{2 N}\right)_{2 i}\right|
$$

in the error tables below.

Assuming convergence of order $\left(N^{-1} \ln N\right)^{r}$ for some $r$, we estimate the classical convergence rate $r$ from

$$
\begin{aligned}
R_{\varepsilon}^{N} & =\frac{\ln \tilde{E}_{\varepsilon}^{N}-\ln \tilde{E}_{\varepsilon}^{2 N}}{\ln \left(\frac{2 \ln N}{\ln 2 N}\right)} \\
& =\frac{\ln \tilde{E}_{\varepsilon}^{N}-\ln \tilde{E}_{\varepsilon}^{2 N}}{\ln \left(\frac{2 k}{k+1}\right)}, \quad \text { for } N=2^{k} \text { and } k=5,6, \ldots, 11 .
\end{aligned}
$$

The last row of each rate table is the uniform convergence rate,

$$
R^{N}=\frac{\ln \tilde{E}^{N}-\ln \tilde{E}^{2 N}}{\ln \left(\frac{2 k}{k+1}\right)}
$$

where $\tilde{E}^{N}=\max _{\varepsilon} \tilde{E}_{\varepsilon}^{N}$.

Example. Consider the following problem of Herceg [7]:

$$
\begin{gathered}
-\varepsilon^{2} u^{\prime \prime}+\left(u^{2}+u-0.75\right)\left(u^{2}+u-3.75\right)=0 \quad \text { for } x \in(0,1), \\
u(0)=u(1)=0 .
\end{gathered}
$$

We have

$$
b_{u}(x, u)=(2 u+1)\left(2 u^{2}+2 u-4.5\right) .
$$

The reduced problem

$$
b(x, u)=0
$$

has four solutions $u_{1}=-2.5, u_{2}=-1.5, u_{3}=0.5$ and $u_{4}=1.5$. It is easy to see that

$$
b_{u}\left(x, u_{1}\right)=-12, \quad b_{u}\left(x, u_{2}\right)=6, \quad b_{u}\left(x, u_{3}\right)=-6 \text { and } b_{u}\left(x, u_{4}\right)=12 .
$$

Hence, $u_{1}$ and $u_{3}$ are not stable reduced solutions of (5.1). A calculation shows that $u_{2}$ and $u_{4}$ satisfy the conditions (1.4). Thus (5.1) is a problem of type (B) with two stable reduced solutions $u_{2}$ and $u_{4}$. Each of $u_{2}$ and $u_{4}$ is "close" (in the sense of Theorem 2.1) to a solution of (5.1) when $\varepsilon$ is sufficiently small. We apply the scheme $\left\{F, X_{s}^{N}\right\}$ to compute these solutions of (5.1). 
TABLE 5.1. Errors for solution near $u_{2}$

\begin{tabular}{|c|ccccc|}
\hline$\varepsilon$ & N=64 & 128 & 256 & 512 & 1024 \\
\hline $2.500000 \mathrm{e}-01$ & $3.4316 \mathrm{e}-04$ & $8.6686 \mathrm{e}-05$ & $2.1679 \mathrm{e}-05$ & $5.4227 \mathrm{e}-06$ & $1.3557 \mathrm{e}-06$ \\
$6.250000 \mathrm{e}-02$ & $3.5179 \mathrm{e}-03$ & $1.1715 \mathrm{e}-03$ & $3.4384 \mathrm{e}-04$ & $8.6876 \mathrm{e}-05$ & $2.1726 \mathrm{e}-05$ \\
$1.562500 \mathrm{e}-02$ & $3.5180 \mathrm{e}-03$ & $1.1715 \mathrm{e}-03$ & $3.7359 \mathrm{e}-04$ & $1.1596 \mathrm{e}-04$ & $3.5084 \mathrm{e}-05$ \\
$3.906250 \mathrm{e}-03$ & $3.5180 \mathrm{e}-03$ & $1.1715 \mathrm{e}-03$ & $3.7359 \mathrm{e}-04$ & $1.1596 \mathrm{e}-04$ & $3.5084 \mathrm{e}-05$ \\
$9.765625 \mathrm{e}-04$ & $3.5179 \mathrm{e}-03$ & $1.1715 \mathrm{e}-03$ & $3.7359 \mathrm{e}-04$ & $1.1596 \mathrm{e}-04$ & $3.5084 \mathrm{e}-05$ \\
$2.441406 \mathrm{e}-04$ & $3.5179 \mathrm{e}-03$ & $1.1715 \mathrm{e}-03$ & $3.7359 \mathrm{e}-04$ & $1.1596 \mathrm{e}-04$ & $3.5084 \mathrm{e}-05$ \\
$6.103516 \mathrm{e}-05$ & $3.5179 \mathrm{e}-03$ & $1.1715 \mathrm{e}-03$ & $3.7359 \mathrm{e}-04$ & $1.1596 \mathrm{e}-04$ & $3.5084 \mathrm{e}-05$ \\
$1.525879 \mathrm{e}-05$ & $3.5179 \mathrm{e}-03$ & $1.1715 \mathrm{e}-03$ & $3.7359 \mathrm{e}-04$ & $1.1596 \mathrm{e}-04$ & $3.5084 \mathrm{e}-05$ \\
$3.814697 \mathrm{e}-06$ & $3.5179 \mathrm{e}-03$ & $1.1715 \mathrm{e}-03$ & $3.7359 \mathrm{e}-04$ & $1.1596 \mathrm{e}-04$ & $3.5084 \mathrm{e}-05$ \\
$9.536743 \mathrm{e}-07$ & $3.5179 \mathrm{e}-03$ & $1.1715 \mathrm{e}-03$ & $3.7359 \mathrm{e}-04$ & $1.1596 \mathrm{e}-04$ & $3.5084 \mathrm{e}-05$ \\
\hline
\end{tabular}

TABLE 5.2. Convergence rates for solution near $u_{2}$

\begin{tabular}{|c|cccc|}
\hline$\varepsilon$ & $\mathrm{N}=64$ & 128 & 256 & 512 \\
\hline $2.500000 \mathrm{e}-01$ & 2.55 & 2.48 & 2.41 & 2.36 \\
$6.250000 \mathrm{e}-02$ & 2.04 & 2.19 & 2.39 & 2.36 \\
$1.562500 \mathrm{e}-02$ & 2.04 & 2.04 & 2.03 & 2.03 \\
$3.906250 \mathrm{e}-03$ & 2.04 & 2.04 & 2.03 & 2.03 \\
$9.765625 \mathrm{e}-04$ & 2.04 & 2.04 & 2.03 & 2.03 \\
$2.441406 \mathrm{e}-04$ & 2.04 & 2.04 & 2.03 & 2.03 \\
$6.103516 \mathrm{e}-05$ & 2.04 & 2.04 & 2.03 & 2.03 \\
$1.525879 \mathrm{e}-05$ & 2.04 & 2.04 & 2.03 & 2.03 \\
$3.814697 \mathrm{e}-06$ & 2.04 & 2.04 & 2.03 & 2.03 \\
$9.536743 \mathrm{e}-07$ & 2.04 & 2.04 & 2.03 & 2.03 \\
\hline$R^{N}$ & 2.04 & 2.04 & 2.03 & 2.03 \\
\hline
\end{tabular}

TABLE 5.3. Errors for solution near $u_{4}$

\begin{tabular}{|c|ccccc|}
\hline$\varepsilon$ & $\mathrm{N}=64$ & 128 & 256 & 512 & 1024 \\
\hline $2.500000 \mathrm{e}-01$ & $1.1820 \mathrm{e}-03$ & $2.9456 \mathrm{e}-04$ & $7.3582 \mathrm{e}-05$ & $1.8397 \mathrm{e}-05$ & $4.5991 \mathrm{e}-06$ \\
$6.250000 \mathrm{e}-02$ & $5.5968 \mathrm{e}-03$ & $1.8000 \mathrm{e}-03$ & $5.6725 \mathrm{e}-04$ & $1.7490 \mathrm{e}-04$ & $5.2884 \mathrm{e}-05$ \\
$1.562500 \mathrm{e}-02$ & $5.6164 \mathrm{e}-03$ & $1.8021 \mathrm{e}-03$ & $5.6737 \mathrm{e}-04$ & $1.7490 \mathrm{e}-04$ & $5.2884 \mathrm{e}-05$ \\
$3.906250 \mathrm{e}-03$ & $5.6057 \mathrm{e}-03$ & $1.8022 \mathrm{e}-03$ & $5.6760 \mathrm{e}-04$ & $1.7493 \mathrm{e}-04$ & $5.2885 \mathrm{e}-05$ \\
$9.765625 \mathrm{e}-04$ & $5.5976 \mathrm{e}-03$ & $1.8008 \mathrm{e}-03$ & $5.6743 \mathrm{e}-04$ & $1.7493 \mathrm{e}-04$ & $5.2888 \mathrm{e}-05$ \\
$2.441406 \mathrm{e}-04$ & $5.5951 \mathrm{e}-03$ & $1.8002 \mathrm{e}-03$ & $5.6731 \mathrm{e}-04$ & $1.7491 \mathrm{e}-04$ & $5.2886 \mathrm{e}-05$ \\
$6.103516 \mathrm{e}-05$ & $5.5944 \mathrm{e}-03$ & $1.8000 \mathrm{e}-03$ & $5.6727 \mathrm{e}-04$ & $1.7490 \mathrm{e}-04$ & $5.2884 \mathrm{e}-05$ \\
$1.525879 \mathrm{e}-05$ & $5.5943 \mathrm{e}-03$ & $1.8000 \mathrm{e}-03$ & $5.6726 \mathrm{e}-04$ & $1.7490 \mathrm{e}-04$ & $5.2884 \mathrm{e}-05$ \\
$3.814697 \mathrm{e}-06$ & $5.5942 \mathrm{e}-03$ & $1.8000 \mathrm{e}-03$ & $5.6726 \mathrm{e}-04$ & $1.7490 \mathrm{e}-04$ & $5.2884 \mathrm{e}-05$ \\
$9.536743 \mathrm{e}-07$ & $5.5942 \mathrm{e}-03$ & $1.8000 \mathrm{e}-03$ & $5.6726 \mathrm{e}-04$ & $1.7490 \mathrm{e}-04$ & $5.2884 \mathrm{e}-05$ \\
\hline
\end{tabular}

The numerical results for the example show that the scheme is capable of computing those solutions of problem (B) that lie close to particular reduced solutions. Furthermore, the scheme achieves second-order accuracy for this difficult problem, confirming our theoretical results. 
TABLE 5.4. Convergence rates for solution near $u_{4}$

\begin{tabular}{|c|cccc|}
\hline$\varepsilon$ & $\mathrm{N}=64$ & 128 & 256 & 512 \\
\hline $2.500000 \mathrm{e}-01$ & 2.58 & 2.48 & 2.41 & 2.36 \\
$6.250000 \mathrm{e}-02$ & 2.10 & 2.06 & 2.04 & 2.03 \\
$1.562500 \mathrm{e}-02$ & 2.11 & 2.07 & 2.05 & 2.04 \\
$3.906250 \mathrm{e}-03$ & 2.11 & 2.06 & 2.05 & 2.04 \\
$9.765625 \mathrm{e}-04$ & 2.10 & 2.06 & 2.05 & 2.04 \\
$2.441406 \mathrm{e}-04$ & 2.10 & 2.06 & 2.04 & 2.03 \\
$6.103516 \mathrm{e}-05$ & 2.10 & 2.06 & 2.04 & 2.03 \\
$1.525879 \mathrm{e}-05$ & 2.10 & 2.06 & 2.04 & 2.03 \\
$3.814697 \mathrm{e}-06$ & 2.10 & 2.06 & 2.04 & 2.03 \\
$9.536743 \mathrm{e}-07$ & 2.10 & 2.06 & 2.04 & 2.03 \\
\hline$R^{N}$ & 2.10 & 2.06 & 2.04 & 2.03 \\
\hline
\end{tabular}

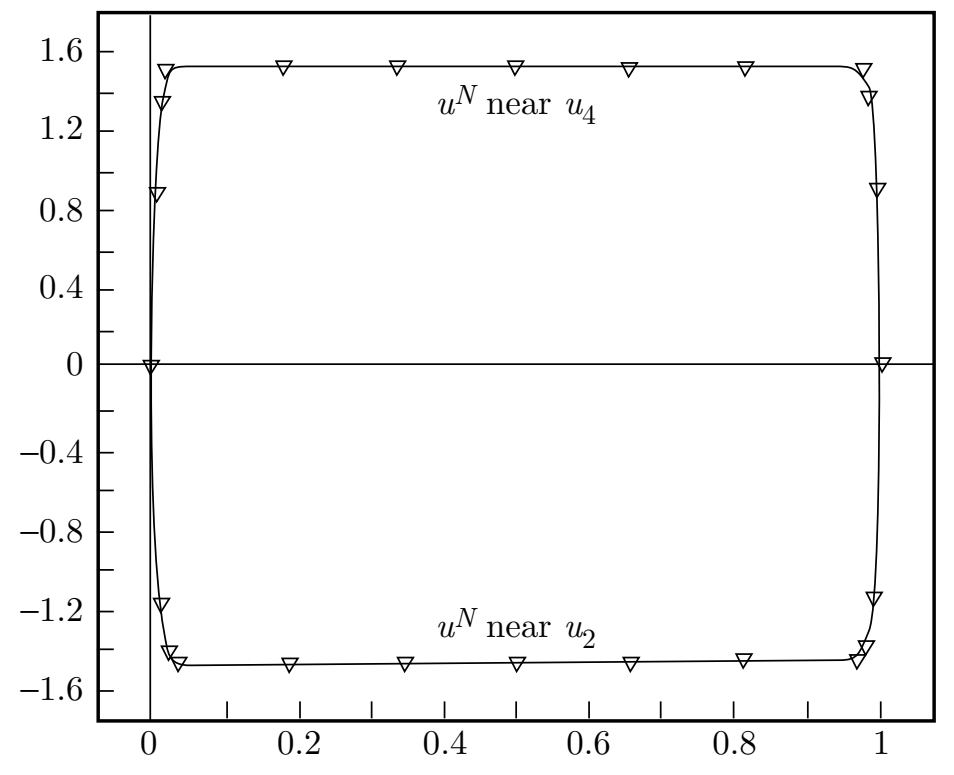

$\nabla$ the computed solution $u^{12}$

- Piecewise linear interpolant of the computer solution $u^{256}$

$$
\varepsilon=1.5625 \mathrm{e}-02
$$

\section{FigURE 1}

In Figure 1 we display the computed solutions of (5.1) that lie near $u_{2}$ and $u_{4}$ when $\varepsilon=1.5625 \mathrm{e}-02$, with $N=12$ (discrete points marked by triangles) and $N=256$ (continuous piecewise linear interpolant to the computed solution). The proximity of the solutions for $N=12$ and $N=256$ demonstrates the accuracy of the method when only a small number of points is used.

Figure 2 shows the behavior of the same problem inside the layer region $0 \leq x \leq$ 0.04. It compares the solution for $N=24$ (discrete points marked by triangles) with the continuous piecewise linear interpolant to the computed solution for $N=256$. 


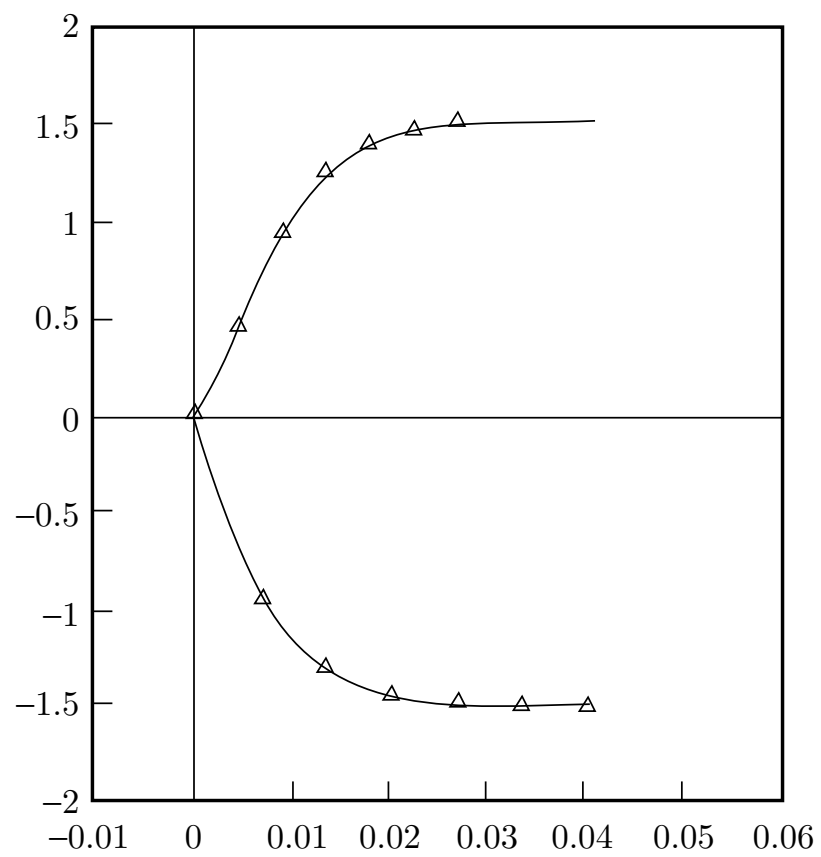

Enlargement of computer solutions near $x=0$; $\Delta$ is $u^{24}$ and the continuous curve is the piecewise linear interpolant of $u^{256}$

FiguRE 2

Clearly, the method tracks the layer accurately even when using relatively few points.

\section{ACKNOWLEDGEMENT}

We thank the referee for encouraging us to improve the original proof of Lemma 2.4 .

\section{REFERENCES}

1. K.W. Chang and F.A. Howes, Nonlinear singular perturbation phenomena, Springer, New York, 1984. MR 86e:34090

2. C.M. D'Annunzio, Numerical analysis of a singular perturbation problem with multiple solutions, Ph.D. Dissertation, University of Maryland at College Park, 1986 (unpublished).

3. E.P. Doolan, J.J.H. Miller and W.H.A. Schilders, Uniform numerical methods for problems with initial and boundary layers, Boole Press, Dublin, 1980. MR 82h:65053

4. P.C. Fife, Semilinear elliptic boundary value problems with small parameters, Arch. Rational Mech. Anal. 52(1973), 205-232. MR 51:10863

5. E.C. Gartland, Jr., Graded-mesh difference schemes for singularly perturbed two-point boundary value problems, Math. Comp. 51(1988), 631-657. MR 89d:65073

6. A.F. Hegarty, J.J.H. Miller and E. O'Riordan, Uniform second order difference schemes for singular perturbation problems, Boundary and interior layers - Computational and asymptotic methods (J.J.H. Miller, ed.), Boole Press, Dublin, 1980, pp.301-305. MR 83h:65095

7. D. Herceg, Uniform fourth order difference scheme for a singular perturbation problem, Numer. Math. 56(1990), 675-693. MR 91f:65137 
8. D. Herceg and N. Petrović, On numerical solution of a singularly perturbed boundary value problem II, Univ. u Novom Sadu, Zb. Rad. Pirod.-Mat. Fak. Ser. Mat. 17 (1987), 163-186. MR 89c:65084

9. J. Lorenz, Stability and monotonicity properties of stiff quasilinear boundary problems, Zb. rad. Prir. Mat. Fak. Univ. Novom Sadu, Ser. Mat. 12(1982), 151-176. MR 85e:34046

10. K. Niijima, On a three-point difference scheme for a singular perturbation problem without a first derivative term I, Mem. Numer. Math., 7(1980), 1-10. MR 82a:65059

11. R.E. O'Malley, Singular perturbation methods for ordinary differential equations, SpringerVerlag, New York, 1991. MR 92i:34071

12. E. O'Riordan and M. Stynes, A uniformly accurate finite element method for a singularly perturbed one-dimensional reaction-diffusion problem, Math. Comp. 47(1986), 555-570. MR 88f: 65126

13. J.M. Ortega and W.C. Rheinboldt, Iterative solution of nonlinear equations in several variables, Academic Press, New York, 1970. MR 42:8686

14. H.-G. Roos, Global uniformly convergent schemes for a singularly perturbed boundary value problem using patched base spline-function, J. Comp. Appl. Maths. 29(1990), 69-77. MR 91d:65108

15. G.I. Shishkin, Grid approximation of singularly perturbed parabolic equations with internal layers, Sov. J. Numer. Anal. Math. Modelling 3(1988), 393-407. MR 89k:65109

16. D.R. Smith, Singular-perturbation theory (an introduction with applications), Cambridge University Press, Cambridge, 1985. MR 87d:34001

17. R. Vulanović, On a numerical solution of a type of singularly perturbed boundary value problem by using a special discretization mesh, Zb. Rad. Prir. Mat. Fak. Univ. Novom Sadu ser. Mat. 13(1983), 187-201. MR 87a:65133

18. R. Vulanović, Exponential fitting and special meshes for solving singularly perturbed problems, IV Conference on Applied Mathematics (B. Vrdoljak, ed.) Split, 1985, pp.59-64. CMP 18:04

Department of Mathematics, University College, Cork, Ireland

E-mail address: stynes@ucc.ie 